ÉGYPTE monde arabe

\section{Égypte/Monde arabe}

3 | 2006

Terrains d'Égypte, anthropologies contemporaines

\title{
Deux ethnologues dans le métro
}

Jean-Charles Depaule et Philippe Tastevin

\section{(2) OpenEdition}

\section{Journals}

Édition électronique

URL : https://journals.openedition.org/ema/1073

DOI : 10.4000/ema.1073

ISSN : 2090-7273

\section{Éditeur}

CEDEJ - Centre d'études et de documentation économiques juridiques et sociales

\section{Édition imprimée}

Date de publication : 31 décembre 2006

Pagination : 23-34

ISSN : 1110-5097

\section{Référence électronique}

Jean-Charles Depaule et Philippe Tastevin, « Deux ethnologues dans le métro », Égypte/Monde arabe

[En ligne], 3 | 2006, mis en ligne le 08 juillet 2008, consulté le 07 juillet 2022. URL : http://

journals.openedition.org/ema/1073; DOI : https://doi.org/10.4000/ema.1073 


\section{Jean-Charles Depaule et Philippe Tastevin}

\section{DEUX ETHNOLOGUES DANS LE MÉTRO}

e métro du Caire, dont la première ligne de type RER a été inaugurée en L1987 et la seconde, intra-urbaine, est en service depuis 1996 et 1999, semblait bien se prêter à une étude anthropologique, non pas parce que, selon I'appréciation de Marc Augé', il serait un miroir grossissant, mais plutôt parce que ce mode de déplacement, importé dans une agglomération où existe une longue tradition de transports privés et publics de toutes sortes, constituait une innovation de taille dans l'existence quotidienne des Cairotes, une innovation technique et, selon ses promoteurs et ses gestionnaires, un dispositif massif devant assurer efficacement des flux en disciplinant ses usagers. Au début des années 2000, suffisamment de temps s'était écoulé pour que l'on essaie de comprendre comment ce corps étranger était entré, à l'évidence, dans les mœurs cairotes. En outre, il était désormais possible, non seulement de comparer le métro aux autres moyens de locomotion collectifs qui l'avaient précédé et qui pour beaucoup continuaient de fonctionner, mais d'observer si chacun des deux types de ligne, le premier de conception française, le second japonais, avait des effets propres sur les comportements.

Revenant, dans ce retour sur notre "cuisine du métro ", sur la double enquête que nous y avons menée, en parallèle pour une large part, nous avons essayé de répondre aux interrogations formulées par les initiateurs de ce dossier, concernant l'observation, la question du local, "I'unité de temps et de lieu » et la description.

\section{OBSERVATION PARTICIPANTE}

Comme le café où il est difficilement concevable qu'il ne soit pas consommateur, le métro est un terrain où l'observateur est nécessairement engagé dans la situation qu'il doit décrire. Sa place - le terme est à entendre ici littéralement, place assise ou debout - n'est donc pas en soi inconfortable puisqu'il est un

1. Augé M., 1986, Un ethnologue dans le métro, Paris, Hachette. 
usager, qui a acquis le droit d'être là en achetant son titre de transport (quelquefois on le lui a offert). Toutefois, s'il est étranger, il est un voyageur d'un genre un peu inhabituel : il est l'objet d'attentions particulières, qui relèvent du registre de l'hospitalité, et d'un traitement spécial, généralement bienveillant, si son comportement n'est pas conforme aux codes partagés. Comme tout utilisateur du métro, il a dû en faire tôt ou tard l'apprentissage en intériorisant des comportements dont la maîtrise le désigne aujourd'hui comme familier. Des inévitables bévues qu'il a commises, dont la plus embarrassante était d'entrer par mégarde dans la voiture réservée aux femmes, il a essayé de tirer les leçons (on le sait, les situations d'embarras ont une vertu pédagogique). Sa situation ainsi acquise devient éventuellement inconfortable, un basculement s'opère et il est perçu comme doublement étranger, en tant qu'étranger au pays et observateur, dès que son regard devient insistant, qu'il sort son carnet et son stylo ou son appareil de photo. Mais ceux-ci pourront être oubliés au bout d'un certain temps, comme l'est la présence d'un ouvrier en train de changer une ampoule au milieu d'une assemblée (merci à Christian Lallier, cinéaste et ethnologue, inspirateur de cette comparaison).

L'observateur voyageur s'est peu à peu constitué une mémoire. Elle est double, en l'occurrence, puisqu'elle est celle des deux observateurs signataires de cet article, partagée à travers les échanges de travail et, plus particulièrement, l'élaboration commune de textes.

Pour l'un, cette mémoire s'est nourrie non seulement de la fréquentation du métro depuis l'ouverture de la première ligne, mais aussi par une pratique de plus de trente ans des divers transports publics de l'agglomération du Caire, y compris certains qui ont disparu, le tramway ou le minibus de Zamalek ou la ligne du train de banlieue vers Hilwân, nommée déjà "métro », comme celle du tramway rapide desservant jusqu'aujourd'hui Héliopolis et appelé désormais al-mêtro al-'âdî, « le métro ordinaire » (par différence avec al-mêtro al-anfâq, le « métro des souterrains »). Restée longtemps assez largement inattentive, cette pratique est devenue relation d'enquête et le métro, " terrain ", d'abord au cours d'une phase préparatoire au début des années 1990, ensuite, à l'occasion de deux séjours, au printemps 2001 et au printemps 2002. L'autre découvrait en 2000, en même temps que le Caire, le métro en « apprenant à le prendre " à travers une utilisation quotidienne. Un apprentissage visuel, à défaut de maîtriser la langue, du moins au début. Pour " l'incompétent du métro » qu'il était alors, l'enquête de terrain consistait, à ses débuts, à tirer profit de son insuffisance initiale. Combiner des déplacements réguliers, accomplis en tant qu'usager parmi les usagers, avec une observation lente et située de leurs interactions, le plus souvent un carnet de notes à la main, lui permettait de construire, par approximations et ajustements successifs, exemples et contre-exemples, ce que I'on pourrait appeler le mode d'emploi du métro, indicateur des convenances et des usages en vigueur.

Pour ces raisons et sans doute pour bien d'autres, dont les plus évidentes sont l'âge, la taille et le cursus, ce que chacun de nous deux regarde diffère, 
ainsi que la manière complémentaire dont, délibérément ou non, nous le faisons, dans le dessein de produire, notamment à travers des descriptions (on en verra un exemple) qui sont comme des compromis d'écriture, une «fiction» plus complexe que ne le serait un texte rédigé par un seul et même auteur.

L'un - désigné ici comme " l'absent » - ne réside plus au Caire, où il se rend épisodiquement. L'autre vit en Égypte. Sa vision est plus immédiate, microsociologique, focalisée sur les petits territoires, les arrangements ordinaires entre les gens et le dispositif de ce « lieu mouvement » (Isaac Joseph²) qu'ils traversent. De semaine en semaine, il prend la mesure de l'histoire en train de se faire, des innovations introduites périodiquement depuis l'ouverture de la ligne $\mathrm{n}^{\circ} 1$. Elles sont non seulement d'ordre technique (sur la ligne $n^{\circ} 2$, escaliers roulants, air conditionné...) mais organisationnelles: par exemple le prix unique du trajet (75 piastres), à la française, a supplanté le système à l'anglaise de tarifs modulés selon la distance, décourageant les trajets brefs et favorisant les plus longs ou, depuis 2005, des flèches rouges et vertes tracées sur le sol des quais et, dans les voitures, des inscriptions sont destinées à discipliner la montée et la descente, moment critique d'un trajet sur lequel nous reviendrons plus en détail. Dans les couloirs l'affichage publicitaire se développe dans des encadrements vitrés et lumineux prévus à cet effet dont beaucoup restent vides. Et en d'autres endroits : sur les contremarches des escaliers qui, vues à une certaine distance, fonctionnent comme des tableaux d' "Op art » composés de lamelles; sur les quais, où des clips vidéos sont également retransmis par des moniteurs; et dans les voitures, en bandeaux au-dessus des fenêtres. Avec la toute récente campagne de I'opérateur de téléphonie mobile Vodafone, on change d'échelle, puisqu'elle transforme entièrement l'aspect extérieur de certaines rames, en en recouvrant les wagons d'un film autocollant rouge vif, couleur emblématique de cette société. L'habillage publicitaire et la modification de la tarification constituent les deux principales mesures de la nouvelle politique commerciale du métro, qui correspond à un changement institutionnel majeur : I'affranchissement de la gestion (rentable) du métro de sa tutelle historique, l'organisme des chemins de fer (déficitaire). Autre nouveauté, dans un registre différent, l'appel à la prière du vendredi dans l'enceinte du métro.

Ainsi se constitue une chronique des changements, généralement menus, qui sont autant de tentatives d'aménagement du cadre tel qu'il a été conçu à l'origine. Elle permet que l'absent mette à jour ses connaissances. Celui-ci a tendance à référer davantage cette chronique aux trois décennies d'une histoire récente et aux évolutions d'une géographie des mobilités intra-urbaines.

L'un et l'autre chercheur s'attachent aux gestes qui contribuent à atténuer la pénibilité des déplacements que chaque usager est amené à accomplir à

2. Joseph I., 1998, La Ville sans qualités, Éditions de l'Aube. 
un moment ou un autre de sa carrière de voyageur. Et, plus précisément, aux circonstances dans lesquelles les marques d'attention à autrui s'expriment. Replacé dans une perspective temporelle plus longue que celle du moment où il s'accomplit, le fait de céder son siège apparaît comme un usage inédit. Il est impossible dans les autobus bondés. En revanche, I'usage pour une personne assise de s'emparer, sans autre forme de procès, d'un enfant en l'arrachant aux bras de sa mère ou de son père, d'un cartable ou d'un cabas des mains de son propriétaire pour le poser sur ses genoux, appartient, de longue date, à une culture des transports cairotes.

Quelques mots sur les vertus et les limites de la pratique du terrain en binôme. D'une expérience sur le tronçon de la ligne $n^{\circ} 1$, entre la station Sadate (Tahrîr) et le terminus d'al-Marg al-Gadîda, se dessinent quelques conclusions. Lorsqu'elle est menée à deux de façon concomitante, I'observation est plus construite, en même temps l'attention est moins flottante, l'un des deux chercheurs attire l'attention de l'autre, propose en cours de route une interprétation, précise ou recoupe une information. Le champ se peuple de détails pertinents supplémentaires. Surtout, l'observation commune autorise, comme l'a noté la cinéaste Emmanuelle Demoris, des degrés d'engagement et de désengagement différentiels. Il est possible de moduler davantage la focalisation du regard, grâce à la redistribution, au gré des circonstances, des tâches. Celles-ci s'avèrent activement complémentaires : parler permet à l'autre de se taire, comme une relance de la conversation lui permet de noter une idée, rester avec un interlocuteur privilégié donne à l'autre la liberté de sortir du champ, pour s'abstraire de propos de pure convenance, prendre une photo, vérifier quelque chose en passant, pour prêter l'oreille aux voyageurs d'à côté, regarder à travers la vitre.

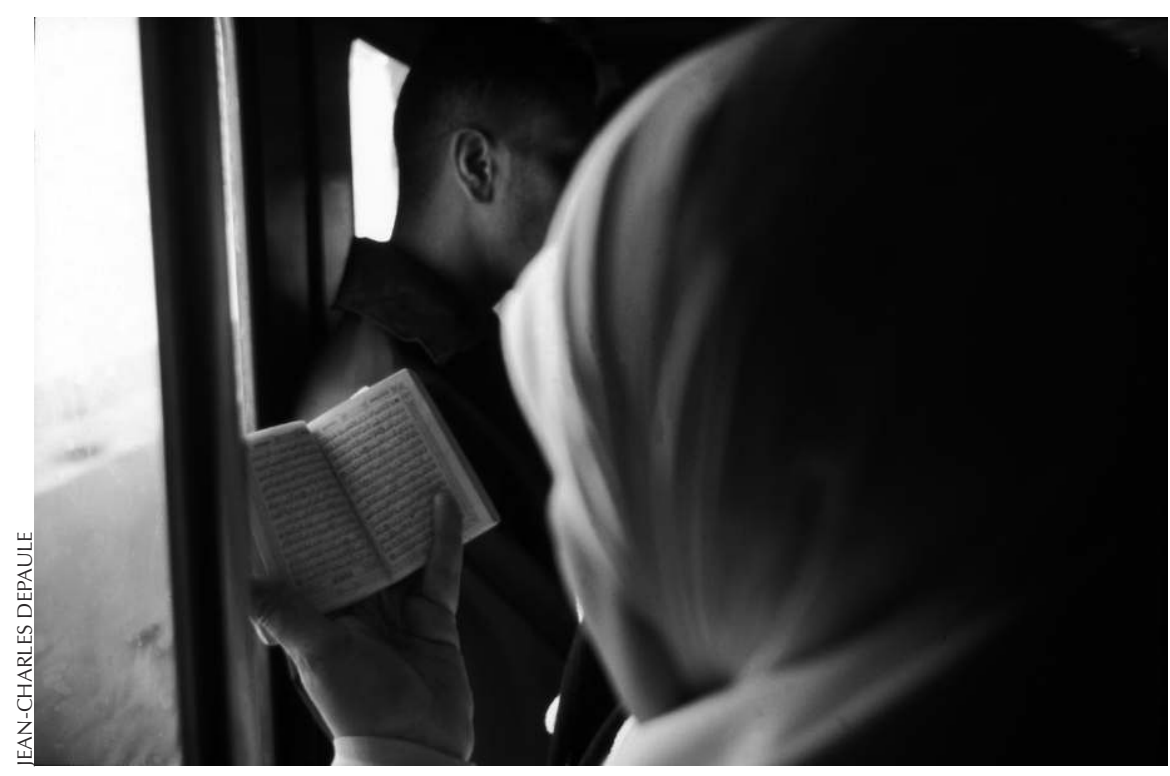


En outre, l'enquête se trouve facilitée s'il faut gérer une crise comme lorsque la présence d'un chercheur est contestée ou, notamment en sollicitant la médiation d'un tiers, surmonter les mille et un embarras qu'il rencontre. On évoquera ici plus particulièrement, dans le contexte où, dans un lieu public, I'un des deux chercheurs prend des photos, le délicat temps mort qu'est inévitablement un changement d'optique ou de pellicule (rembobinage, ouverture du boîtier, déchargement, rechargement, réamorçage), de batterie ou de carte mémoire dans le cas d'un appareil numérique : une brèche s'ouvre pour des commentaires, questions, objections jusque-là contenus, de la part des témoins proches qui interfèrent. Du coup, pris, voire empêtré dans des opérations techniques accessoires qui le désarment, l'observateur photographe est mis sur la sellette, n'est plus oublié. La présence d'un compagnon de terrain permet de conjurer cette surexposition momentanée en maintenant l'intensité de l'interaction et du regard, en donnant s'il le faut un supplément d'explication. Dans la division du travail en train de se faire, lui revient la part de vigilance qui garantit ou au besoin rétablit une nécessaire marge de manœuvre. Elle ne s'exerce pas seulement dans la situation qui vient d'être décrite, inconfortable pour le photographe qui se sent vulnérable, mais dans l'ensemble des opérations de la prise de vue.

De façon générale, l'observation participante conjointe tend à légèrement se dédoubler, sans que les rôles soient fixés une fois pour toutes : le plus souvent elle est, alternativement, plus participante pour l'un, plus observatrice pour I'autre.

\section{LA QUESTION DU LOCAL}

D’une façon générale, au Caire sans doute plus encore qu'ailleurs, les habitants ont tendance à décloisonner les espaces et moments de leur vie quotidienne. Dans des lieux plus contraints que leur domicile et ses abords, plus spécialisés du point de vue fonctionnel et offrant à leurs occupants une marge d'initiative et de liberté (le travail, les transports...) en principe limitée, ils introduisent des activités et des valeurs qui sont autant de "morceaux » qui semblent ressortir plutôt à d'autres domaines de leur existence. L'image du fonctionnaire extrayant du tiroir de son bureau un sandwich ne surprend pas les familiers des administrations égyptiennes. Ra'fat al-Mihi, réalisateur du film L'Avocat (avec Adel Imam, 1984) a présenté un cas si limite, mais plausible, dans sa visée satirique, qu'il a choqué de nombreux spectateurs, en montrant une femme qui, en faisant la classe à ses élèves, épluche des haricots (ou fait la classe en épluchant des haricots?). On ne s'étonnera donc pas que, dans le métro du Caire, les voyageurs lisent (des journaux, des livres scolaires ou non, et le Coran, psalmodié à mi-voix ou lu silencieusement), qu'ils prient, qu'ils révisent leurs leçons et fassent leurs devoirs, que des femmes tricotent ni que certains profitent de l'attente dans une station à ciel ouvert pour acheter pour plus tard un sandwich à 


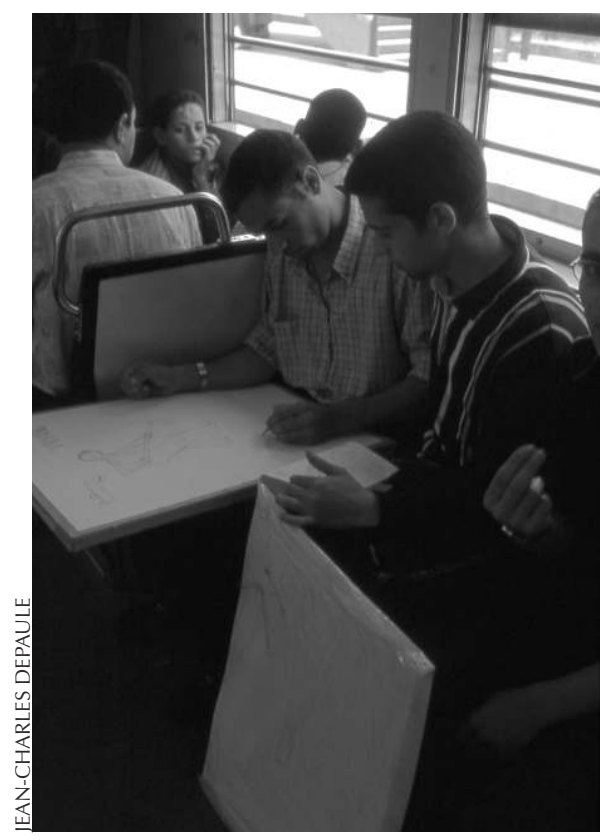

un marchand ambulant, à travers les grilles qui séparent le quai du reste de la ville, comme ils le feraient au coin de la rue, à l'entrée de leur lieu de travail ou à la sortie de l'école. En revanche, pas plus qu'on n'y boit, on $n^{\prime} y$ mange et on $n^{\prime} y$ grignote guère.

Il est possible d'identifier des manières qui relèvent $d^{\prime}$ un savoirvivre commun et prolongent les convenances partagées dans l'espace de I'habitation et de son environnement immédiat, ou les projettent dans I'espace social mobile du métro. II s'agit de ne pas franchir la limite de ce qui est de l'ordre du nizâm ( " l'ordre »), des hagât kwayyisa ( les choses bien ») et nadîfa ( propres ») et de ce qui est tenu pour qalîl aladab (« impoli »). De respecter les personnes âgées, que des voyageurs assis déchargent de leurs paquets le temps du trajet, et I'honneur des femmes, de contrôler le niveau des voix et de veiller à la propreté de l'espace. Si l'on veut prendre la mesure des civilités et de ce qui est considéré comme déplacé, avec toutefois une certaine tolérance pour la turbulence de jeunesse, il est donc, nous semble-t-il, difficile de ne pas référer à la sphère domestique.

Tel est I'un des paradoxes du métro. D'une part, il est une parenthèse mobile (nous insistons sur ce caractère) dans l'espace urbain et dans l'emploi du temps de chaque voyageur. À ce titre, il est justiciable d'analyses hyper-localisées recourant éventuellement à des descriptions microscopiques, jamais complètement arrêtées, dont les limites sont celles de la durée propre et de la dimension spatiale des chaînons successifs à travers lesquels se construit, pour un sujet qui se déplace, la trajectoire qu'il effectue de la sorte dans le grand espace urbain (nous y reviendrons). Mais, d'autre part, son usage ne peut être envisagé indépendamment d'autres pratiques qui relèvent d'autres lieux et d'autres moments. Sans cette mise en perspective, on risquerait de reproduire le type réducteur d'interprétation, strictement disciplinaire, que les technocrates et certains chercheurs labellisés continuent d'avancer, en imputant à l'encadrement, à la panoplie des règlements et des sanctions qui les accompagnent, dont nous ne minimisons pas pour autant l'efficacité, tout le succès du métro qui est comme une oasis miraculeuse d'ordre, de propreté et de relatif silence dans le grand désordre du Caire.

Il est comme une parenthèse "poreuse »... La perméabilité des sphères quotidiennes, ou plutôt, dans ce cas, les transferts d'usages et de codes de la 
sphère domestique (l'habitation et le voisinage) à celle d'un transport public ne sont pas seulement imputables au fait que, par définition, le métro met matériellement en relation à travers l'espace de l'agglomération différents lieux et moments, caractérisés par leur hétérotopie - qu'une approche plus macroscopique est capable d'appréhender.

\section{UNITÉ DE TEMPS ET DE LIEU?}

Au risque de détourner la formule, précisons que nous avons affaire à des unités de découpage, dont la pertinence ne soulève pas de problèmes majeurs, mais qui ne peuvent jamais être envisagées isolément de manière durable.

Le métro considéré dans sa globalité, avec son site propre, l'étendue de son réseau, ses interconnexions, ses équipements, ses multiples dispositifs de surveillance, s'est inséré dans l'ensemble des éléments structurants de l'espace de l'agglomération qu'il concourt à recomposer profondément. Sa consistance est justiciable de descriptions spécifiques notamment au moyen de diagrammes (mode de représentation des flux), de cartes et de plans (mode de représentation de la ville qui a fait pour la première fois son apparition sur la voie publique à l'extérieur et l'intérieur des stations et dont on ne mesure pas encore les effets sur le schéma urbain des Cairotes). Il est formé des multiples agencements d'entités spatiales qui sont les unités privilégiées d'une observation ethnographique : les abords, les accès, la station, ses couloirs, ses guichets, ses barres de péage, ses quais, et puis les rames et leurs voitures du moins celle où le voyageur fulân (quelconque) prend place; et ce qui n'est pas vu du public, que I'on entrevoit à l'occasion, où l'on ne pénètre qu'avec une autorisation spéciale ou à la suite d'une infraction qui amène le fautif à connaître l'ensemble du système pyramidal de contrôle policier de chaque station, des quais jusqu'au bureau de l'officier - par exemple prendre une photographie dans l'enceinte du métro, activité interdite (I'infraction, tactique de terrain?). Mais, avec son administration, ses services techniques et son personnel, ce cadre physique (spatial) a pour vocation, on le sait, la mobilité. Indissociable de durées, de périodicités, il recèle une dimension éminemment temporelle.

L'ethnologue essaie pour sa part de saisir dans leur réalité collective des situations, unités composées à la fois d'espace et de temps. Pas plus que pour les voyageurs qu'il côtoie le temps d'un trajet, cette réalité n'est pour lui peuplée de quantités abstraites ou de masses fluctuantes, mais de visages et de corps en mouvement, dont certains ont, à défaut d'un nom connu, une identité. En suivant ou en reconstituant des trajectoires individuelles et, éventuellement, en les parcourant à son tour, il apprend, en outre, que pour la plupart le voyage ne commence ni ne prend fin avec la séquence de stations parcourues dans le métro, mais, le plus souvent, emprunte aussi un autobus, un microbus, un taxi et, toujours, la marche à pied. Ces enchaînements s'inscrivent dans des 


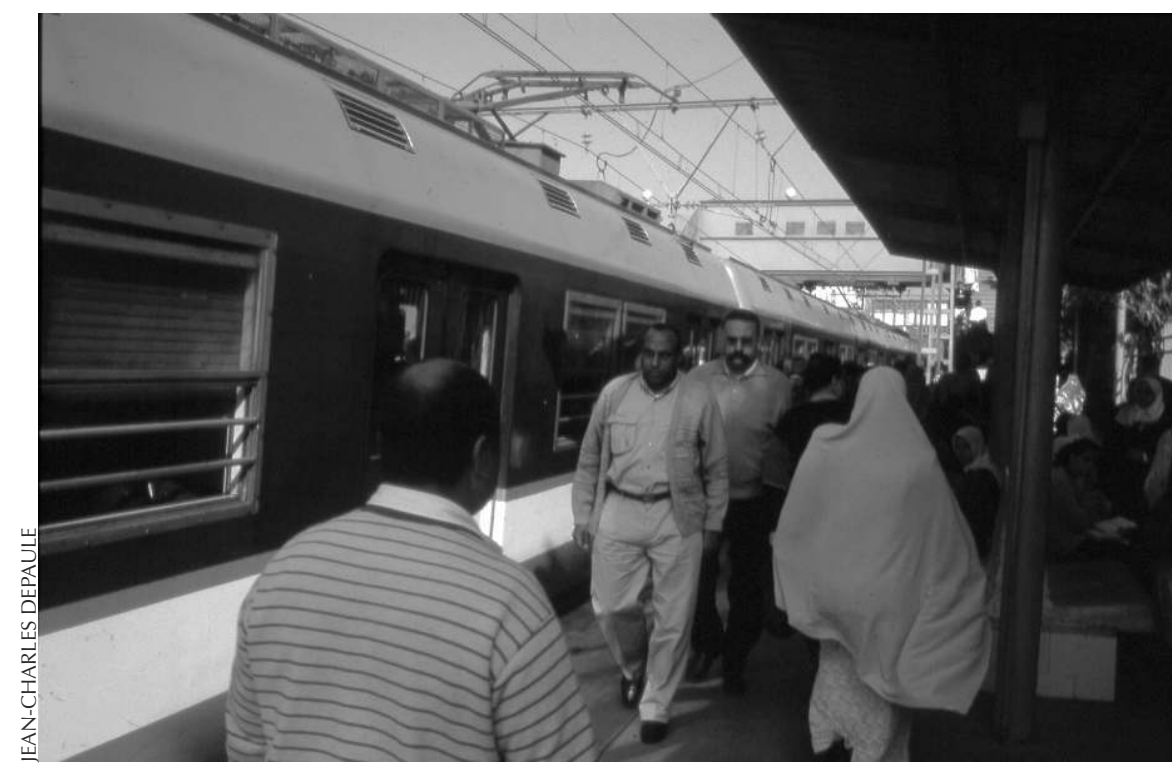

rythmes collectifs, dont ils sont comme autant de variations, et qui appartiennent eux-mêmes aux grands rythmes de la ville.

À la question qui se posait à nous - comment appréhender le mouvement, comment, où et quand? - nous avons essayé de répondre en considérant chaque lieu du point de vue de ce qui s'y déroule et chaque moment du point de vue de sa configuration spatiale. En tentant par exemple de saisir comment, en situation, se tissent l'espace du guichet et le temps de la file d'attente.

\section{DÉCRIRE}

La description ethnographique tente de rendre compte de cette double dimension, temporelle et spatiale. À côté de relevés écrits ou dessinés, d'inventaire d'objets, elle procède essentiellement par séquences et enchaînements. Ont été sélectionnées ici la descente et la montée du train. Le passage des voitures aux quais et des quais à la voiture, qui est au cœur de l'expérience quotidienne du métro, cristallise les représentations d'une foule " anarchique » dans l'espace ordonné métropolitain. Il a d'ailleurs suscité un clip didactique diffusé sur les quais. On risquerait de ne retenir a priori que l'image de forces aveugles se heurtant frontalement, dans une précipitation que la sonnerie annonçant la fermeture automatique des portières dramatise - et cette fatalité de la « masse indisciplinée » détermine encore le choix des gestionnaires dans les innovations nécessaires à la décongestion des rames. À I'inauguration de la première ligne, des rumeurs circulaient : ces " animaux » (hayawânât) seraient incapables d'apprécier à sa juste valeur la modernité du métro. L'indiscipline régnant, ils allaient s'accrocher aux portes comme ils se 
"vautrent » dans l'utubîs. Le métro ne fonctionnerait jamais dans ce pays. Dix ans plus tard, les mêmes préjugés sévissent dans les hautes sphères de I'administration du métro, alors que les voitures atteignent un niveau limite de saturation. L'idée de mettre en place des wagons à deux étages « comme à Paris » pour désengorger la ligne n'a pas été retenue, entre autres raisons parce que le peuple égyptien n'aurait pas la discipline nécessaire à la sécurité de montées et de descentes «fluides » : la voiture à deux étages ne fonctionnerait jamais au Caire.

Une observation plus fine du chevauchement de montées et de descentes, moment caractéristique au cours duquel les territoires du moi sont particulièrement vulnérables, décèle des agencements immanents qui corrigent l'impression première de désordre, de brutalité. Dans cette phase, comme on va le voir, se déploie toute une courtoisie associée à un ensemble de «manœuvres » destinées à pallier la brièveté extrême de l'arrêt de la rame dans la station et la faible capacité de débit de chaque double porte coulissante.

Le goulet d'étranglement est physique et temporel. Au fil des années, la rotation des trains n'a cessé de s'accélérer. Longtemps une rame arrivait à quai environ toutes les huit minutes, les normes d'exploitation des chemins de fer commandaient alors la gestion du métro. Depuis, il aura fallu faire face au succès du nouveau moyen de transport et répondre à une affluence toujours croissante, en augmentant la cadence et le nombre de voitures par rame. Aujourd'hui, chacune en comprend neuf et l'alternance des trains à quai connaît un écart de trois minutes trente secondes en moyenne. Ces données techniques nous intéressent, elles ont des implications directes dans le moment que nous allons décrire. Nous savons qu'à peu près deux millions de personnes - c'est un ordre de grandeur - empruntent le métro quotidiennement, ce qui laisse imaginer la densité des flux «montants » et "descendants », aux heures de pointe, dans les stations centrales.

Le point de vue que nous avons adopté ici est d'abord celui de la descente, séquence dans laquelle l'exigence de coordination prend toute son ampleur, suivra celle de la montée.

Les enjeux de la descente sont plus cruciaux que ceux la montée qui n'est pas son exact symétrique inverse : on laisse sans trop de dommage passer une rame à laquelle on n'a pas pu ou pas su accéder tandis que manquer sa destination a des conséquences plus lourdes.

Sur les quais, en aval, dans l'attente du train, la foule se répartit en longueur avec une inflexion notable en tête de train où l'on trouve les voitures réservées aux femmes. À cet endroit, l'attente piétinante des hommes bute sur une nouvelle borne, elle-même mouvante, personnifiée par un appelé en uniforme, le shâwîsh. Il veille sur l'exclusivité féminine des montées et descentes. Ainsi l'accès aux quais est-il fragmenté, il conditionne fortement les itinéraires de dégagement de la foule qui converge majoritairement à l'autre bout du quai. Une rumeur persistante prétend que les voitures de l'arrière-train sont largement moins bondées (mâ fish zahma). 
Aux heures de pointe, en amont, dans la voiture en marche, les individus qui s'apprêtent à descendre anticipent et se mettent progressivement en place. Compte tenu de l'accessibilité des portes de sortie depuis les différents endroits qu'ils occupent dans la voiture et du degré d'encombrement de chacun, une préparation est nécessaire. Elle opère le passage de l'action individuelle à l'action en commun, hormis la contrainte pressante de temps, et se doit, dans cet effort de coordination, d'assimiler les injonctions liées à la densité des corps en mouvement, à l'ergonomie de l'espace étriqué. Le ballet perpétuel des ajustements est rythmé par les arrivées en station. Un cycle débute toujours à mi-chemin entre deux arrêts avec le mouvement né des interstices qui permettent le dégagement des personnes gênantes parce qu'elles n'appartiennent pas au regroupement des "descendants»: les personnes assises se lèvent, enjoignant d'autres de s'asseoir à leur tour sur le siège qu'elles viennent de libérer. Elles s'amassent devant la porte, en s'assurant par une ritournelle que le dos qui $\mathrm{s}^{\prime}$ interpose ne constitue pas un obstacle :

- Nâzil illi gây? (Vous descendez à la prochaine?)

- In châ' Allah! (Si Dieu le veut!)

En cas de réponse négative, la personne est sommée de dégager la place. Ainsi les gens sont-ils prêts à descendre le plus rapidement possible. La descente en elle-même ressemble à une grosse bousculade, or la clé de cette exigence de coordination réside précisément dans cette précipitation. Finalement, qu'est-ce que cet "agrégat », sinon des individus qui répondent ensemble à l'exigence de l'épreuve en renonçant d'un commun accord aux prérogatives morales? : le corps à corps d'une descente échappe aux présomptions et à l'offense - mais dans la voiture des femmes, la situation de presse et la promiscuité qu'elle implique n'exercent pas la même contrainte que dans celles dont la fréquentation est mixte. C'est pourquoi lors de ce moment précis, hommes ou femmes s'empoignent et s'attrapent, se frottent et s'écartent, se poussent et se tirent, sans jamais s'empêcher, sans que presque jamais un esclandre éclate. Cependant, tant qu'ils sont possibles, des gestes plus discrets, légers, respectueux, somme toute, sont utilisés, en particulier une légère touche sur l'épaule ou le bras du voyageur qui ne vous voit pas, destinée à lui signifier que vous souhaitez vous rapprocher de la porte pour descendre.

Tandis que I'usager, qui entre dans la voiture, remonte le flot en s'aidant de la force du courant inverse, Ego n'attend pas qu'Alter soit descendu, leur action est simultanée, I'un s'appuyant sur l'autre et réciproquement selon la densité des flux. Néanmoins, on n'est pas à l'abri d'incidents; même si normalement la rame ne peut redémarrer que lorsque toutes les portes sont closes, il arrive qu'elles se referment sur une partie du corps, qu'un sac reste suspendu à l'extérieur, qu'un groupe d'individus qui se déplacent ensemble éclate à contrecœur au moment de franchir le seuil.

Ainsi sur la ligne $n^{\circ} 2$, le cas d'une famille qui se scinde entre les deux voitures de tête. Les femmes pénètrent dans le " sanctuaire » féminin avec leurs enfants en bas âge tandis que les hommes montent dans la voiture suivante. 
Mais un petit garçon en pleurs est resté sur le quai. Le shâwîsh intervient, commande l'arrêt de la rame, à la réouverture des portes une femme se saisit de l'enfant. Sur la ligne $n^{\circ} 2$, des fenêtres en bout de voiture (elles sont plus étroites sur la ligne $n^{\circ} 1$ ) assurent d'un wagon à l'autre une continuité permettant une visibilité mutuelle. De part et d'autre des vitres, la famille séparée se recompose provisoirement et échange pendant le reste du trajet les signes d'une frayeur soulagée.

Cette scène a été vue par I'un des deux ethnologues voyageurs, qui I'a ensuite relatée à son collègue. En fonction des questions que celui-ci lui a posées - en faisant notamment appel à des expériences comparables - et de ses demandes de précision, il en a infléchi la brève restitution. Et il a en modifié certaines formulations, en vertu du compromis d'écriture précédemment évoqué.

S'il ne s'agit plus, avec une telle description, même provisoire, du moment du terrain stricto sensu, celui-ci, assurément, ne se limite pas au temps passé sur le terrain, pas plus que l'écriture, comme on l'imagine parfois, ne commence dans une phase ultérieure, celle d'une hypothétique rédaction ( il me reste à rédiger »..., dit-on souvent). Plus encore, sans doute, que dans un travail engageant un seul chercheur, écrire participe de la saisie et de la (re)construction du sens des situations que l'enquête cherche à appréhender et à comprendre, dans un mouvement de va-et-vient avec l'observation.

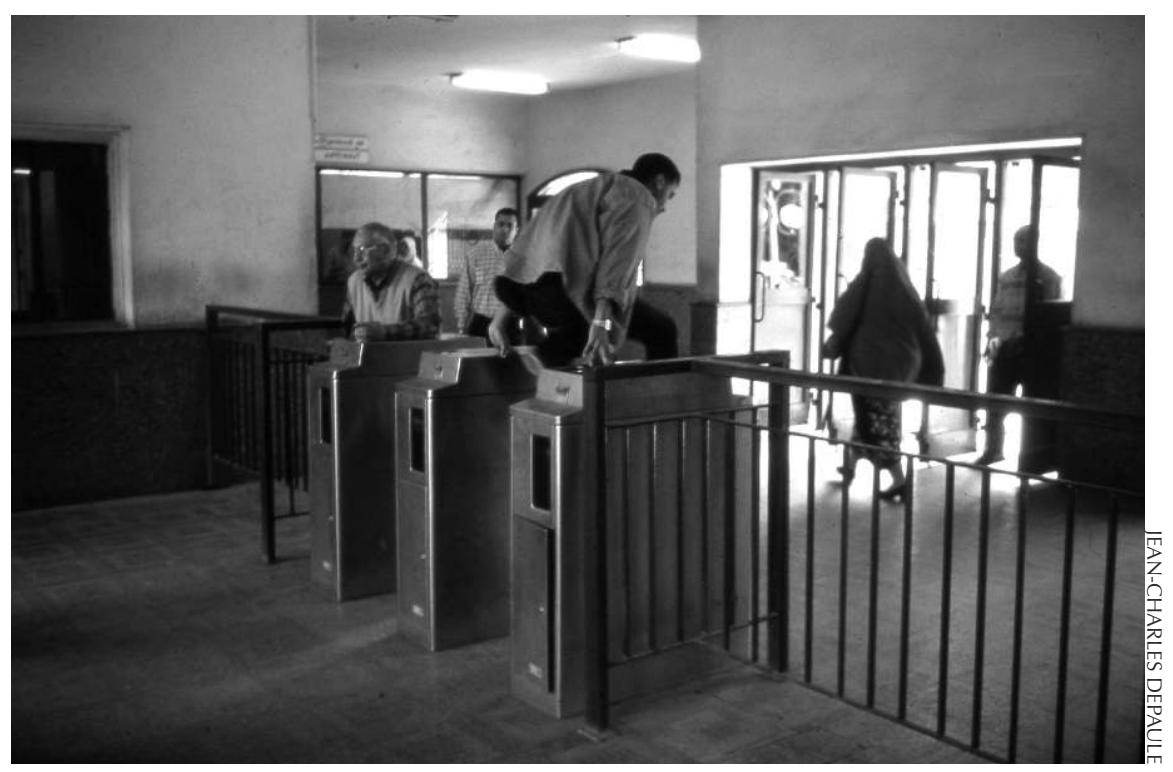

